\title{
Sex Differences in musculoskeletal pain in older adults
}

\author{
Suzanne G. Leveille ${ }^{a}{ }^{*}$, Yuqing Zhang ${ }^{b}$, William McMullen ${ }^{c}$, Margaret Kelly-Hayes ${ }^{d}$, and \\ David T. Felson ${ }^{b}$ \\ a Division of General Medicine and Primary Care, Department of Medicine, Harvard Medical School, Beth \\ Israel Deaconess Medical Center, 330 Brookline Avenue, RO-103, Boston, MA 02215, USA \\ b Arthritis Center, Boston University School of Medicine, Boston, MA, USA \\ c Research and Training Institute, Hebrew Rehabilitation Center, Boston, MA, USA \\ d Department of Neurology, Boston University School of Medicine, Boston, MA, USA
}

\begin{abstract}
Little is known about sex differences in musculoskeletal pain in older persons. There were 682 women and 380 men aged 72 years and older who participated in the 22nd biennial exam of the Framingham Study (1992-1993). Participants were asked to identify pain locations on a homunculus showing all regions of the body. Pain was categorized according to number of regions, with the most disseminated pain classified as widespread pain (back pain and upper and lower extremity pain with bilaterality). Among the women, $63 \%$ reported pain in one or more regions, compared to $52 \%$ of men. Widespread pain was more prevalent among women than men (15 versus $5 \%$, respectively). In both men and women, pain was associated with fair or poor self-rated health, history of back pain before age 65 , and disability. Factors associated with pain only in women included body mass index, systolic blood pressure, and depressive symptoms. In men but not women, pain was associated with polyarticular radiographic osteoarthritis. In conclusion, musculoskeletal pain was more prevalent and more widespread in older women than older men. Men and women differ in the factors associated with musculoskeletal pain in older ages. Further research is needed to understand sex differences in musculoskeletal pain the older population.
\end{abstract}

\section{Keywords}

Pain; Musculoskeletal; Aged; Sex differences; Female; Epidemiology; Arthritis; Obesity

\section{Introduction}

Musculoskeletal joint symptoms affect more than half of older persons in the US (Centers for Disease Control, 2002). In general, older women have a higher prevalence of pain than older men (Helme and Gibson, 2001) and studies suggest that musculoskeletal pain is more disabling for women than men in old age (Hughes et al., 1994; Scudds and Robertson, 1998). Arthritis, which afflicts more women than men (Felson et al., 1995b; Zhang et al., 2003) is a major contributor to joint pain in older adults, but epidemiologic studies have found that pain from arthritis rather than other pathology related to the disease is the primary cause of disability from arthritis (Hughes et al., 1994; Jordan et al., 1997; McAlindon et al., 1993). Additional evidence has shown that physician assessment of joint impairments and radiographs were not 
more informative than self-reported pain in predicting disability (Hughes et al., 1994; Odding et al., 1998, 2000).

In recent years, it has become more clear that location and severity of pain are important determinants of disability in older adults (Lichtenstein et al., 1998; Mobily et al., 1994; Scudds and Robertson, 2000). Many who have chronic musculoskeletal pain, have pain in several sites. For example, in the Women's Health and Aging Study (WHAS), half of women who reported severe foot pain reported pain in at least four other musculoskeletal sites (Leveille et al., 1998). The multi-site nature of pain in the older population has been an obstacle for research because of difficulties in classifying multi-site pain. Summing the number of pain locations or identifying the single site of most severe pain may or may not be relevant for a global problem such as disability.

Using a new classification, based on constellations of musculoskeletal pain, Leveille and colleagues identified widespread musculoskeletal pain as the most disabling pain among older women (Leveille et al., 2001). The classification of widespread pain, based on established criteria, included pain in the upper and lower extremities and axial pain (Wolfe et al., 1990). Population-based studies have shown that the prevalence of widespread pain increases with age, peaking in the seventh and eighth decades (Croft et al., 1993; Wolfe et al., 1995b). Generally, women have a higher prevalence of chronic widespread pain than men, well into late life. Many questions remain to be answered about sex differences in chronic pain in late life, a time when comorbidity is common and accumulating hazards often lead to loss of independence.

Commonly observed differences between men and women in the prevalence and consequences of pain remain unexplained. Although research indicates that pain perception factors, such as menstrual cycle fluctuations in pain sensitivity, may help to explain sex differences in pain reporting in younger adults (Riley et al., 1999), little research has examined sex differences among elders. As a first step toward uncovering clues to sex differences in the experience of pain in old age, we examined pain prevalence and associated risk factors in older men and women in the Framingham Study.

\section{Methods}

The Framingham Heart Study began in 1948 as a longitudinal population-based cohort study of the causes of heart disease. Initially, 5209 men and women between the ages of 30 and 60 years living in Framingham, Massachusetts were enrolled. Biennial examinations were conducted by trained research staff at the study clinic located in Framingham. By the time of the 22nd biennial exam, the cohort included 1166 survivors who ranged in age from 72 to 99 years. The analyses presented here used data from the 22 nd exam because it was the first to collect comprehensive information about musculoskeletal pain. There were 104 persons with missing pain information who were not included in this study. Table 1 presents the demographic and health characteristics of those with non-missing versus missing pain information.

Compared to persons with non-missing pain information, those with missing information were much older, more likely female, cognitively impaired and disabled, and were thus more likely to be examined at home instead of in the clinic where the pain questions were administered. Prevalence of radiographic arthritis was similar in the two groups (x-rays were obtained for persons examined at home or in the clinic). The majority of those with missing information were residing in nursing homes.

The measure of pain used in this study was based on location of frequent pain. During the exam, participants were asked if they had pain, aching or stiffness in any of their joints on most days. Persons who responded, 'yes' were asked to mark painful joints on a homunculus with 
circles showing upper and lower extremity joints, and four areas of the back and neck, and additional diagrams of both hands (three joints on each finger and thumb) and both feet (joints at the base of each toe). In a previous Framingham analysis, more persons reported pain when viewing the homunculus than when asked about pain in the same sites (Edmond and Felson, 2000). Drawing on earlier research showing that number of pain regions predicted worsening disability and falls in older women, pain was categorized according to number of pain regions (Leveille et al., 2001, 2002). Using this method, the most severe category of pain was widespread pain, defined according to the American College of Rheumatology criteria as pain above the waist, below the waist, on both sides of the body, and in the axial region (Wolfe et al., 1990). Pain above the waist, or upper extremity pain, was defined as pain in shoulder, elbow, wrist, thumb, or fingers. Pain below the waist, or lower extremity pain, was defined as pain in the hip, knee, ankle, or base of toes. Contra-lateral upper and lower extremity pain, for example, pain in the right knee along with pain in any site of the left leg or arm, met criteria for widespread pain. Three other categories of pain in these analyses were pain in two to three regions that did not meet criteria for widespread pain, pain in one region, and no pain.

Several characteristics and health and behavioral factors were assessed. Demographic factors included age, sex, education, and marital status. Health factors included measured height and weight, physical activity, functional status, and self-rated health. Body mass index (BMI) was calculated as the ratio of weight in kilograms to height in meters squared. Overweight was classified as BMI>27. Total alcohol consumption was calculated as total ounces per week of beer, wine, and liquor, using methods reported previously (Felson et al., 1988, 1995a). The cutoff for moderate alcohol intake ( $>4$ ounces per week) used in this study approximated the highest quintile. Depressive symptoms were determined by a score of 16 or greater on the wellvalidated Center for Epidemiologic Studies—Depression Scale (CES-D) (Radloff, 1977). Current self-rated health was assessed using the standard 5-level rating, then dichotomized for this study as fair or poor versus good, very good, or excellent. History of back pain was assessed by asking about persistent back pains 'at any time in your life', referring to five age groups from childhood to age 65 and older. The cutpoint of 24 in the Mini-Mental State Examination (MMSE) was used to identify cognitive impairment. The MMSE is a valid and reliable instrument that has been used extensively to screen for cognitive impairment in older populations in research and in clinical practice (Folstein, 1975).

Knee osteoarthritis (OA) was ascertained using anteroposterior weight-bearing radiographs and defined as Kellgren/Lawrence grade 2 or higher in either knee. Hand osteoarthritis was defined as radiographic OA (Kellgren/Lawrence grade>2) in any of 15 hand joints on either hand. The methods of OA ascertainment have been described in detail previously (Zhang et al., 2001, 2003). Blood pressure was measured as the average of two readings conducted by a physician using a mercury column sphygmomanometer (Vasan et al., 2001).

Disability was assessed in two domains: activities of daily living (ADL) and lower extremity mobility. Self-reported need for help from other persons or inability to perform ADL included bathing, dressing, transfers, eating, and using the toilet. Lower extremity mobility disability included self-reported inability to walk a mile and climb stairs without help.

\subsection{Statistical analysis}

Descriptive statistics, mean and percentages, were used for showing the relationships between pain categories and demographic and health characteristics. Age-adjusted prevalence of pain was calculated using the direct standardization method with prevalence applied to the age distribution of the entire cohort according to 5-year age groups. Tests for linear trend across categories of pain constellations were performed using the Mantel-Haenszel chi-square tests ( 1 degree of freedom, d.f.) for categorical variables, and the $F$-statistic ( 1 d.f.) from linear regression models for continuous variables. 
Odds ratios and $95 \%$ confidence limits were derived using multivariate logistic regression methods in sex-stratified models examining risk factors for widespread pain compared to no pain. In the modeling, we included all variables that were significantly associated with pain in the linear trend analyses described above. We used data from the entire cohort to examine sex differences in risk for widespread pain, performing a series of extended logistic regression models to determine the impact of potential confounders on the odds ratio for widespread pain in women compared to men.

\section{Results}

Women had higher age-adjusted prevalence of musculoskeletal pain compared to men, with $64 \%$ of women and $52 \%$ of men reporting pain (Fig. 1). There was a marked difference between women and men in the proportions that had widespread pain, 15 and 5\%, respectively, but similar proportions of women and men reported regional pain and multi-site pain that did not meet criteria for widespread pain.

There were several differences between women and men in factors associated with pain (Table 2). In women, the number of regions of pain was associated with higher BMI, higher systolic blood pressure, and depressive symptoms.

Weight differences in the women were most striking between those with widespread pain compared no pain, with 60 versus $42 \%$, respectively, having a BMI of 27 or higher. There was no trend for higher BMI with more pain in men, though $58 \%$ of men with multi-region pain (not widespread) were overweight compared to $41 \%$ of those without pain. Among men with widespread pain, $40 \%$ were overweight, similar to those with no pain. Although higher proportions of men had depressive symptoms across pain categories, the trend was not significant. Average age did not differ across pain categories in either men or women, though women with widespread pain were slightly older than their peers with other pain or no pain.

The notable difference between women and men in the proportions that were married, related to sex differences in life expectancy leading to greater widowhood in women in old age, was not associated with pain status. Education level was not significantly associated with pain in men or women but there was a somewhat lower proportion of women that completed high school among women with widespread pain compared to other women.

Alcohol consumption was not associated with more pain in women, but men with single or multi-site pain, but not widespread pain, were more likely to consume higher amounts of alcohol than men without pain. In both men and women, those with widespread pain reported the lowest alcohol consumption. In men, but not in women, pain was associated with polyarticular radiographic osteoarthritis (present in at least one knee and one hand joint).

Pain was strongly associated with fair or poor self rated health in both women and men (trend $P$-value $<0.001)$. Nearly half of women with widespread pain reported fair or poor health compared to $12 \%$ of those without pain. Among men, about one-third of those with widespread pain reported fair or poor health compared to $18 \%$ of men with no pain. In both women and men, there was a strong trend across pain categories in the proportions that reported having back pain before age 65 . Similarly, pain level was strongly associated with disability in ADL or lower extremity mobility. Nearly two-thirds of women with widespread pain reported disability compared to $22 \%$ of those with no pain. Men with multi-site pain generally had more disability than those with single site pain or no pain.

Because the strongest differences in prevalence of risk factors were observed between persons with widespread pain and those with no pain, we compared these two categories to determine the magnitude of the differences in women and men using multivariate analyses. Women who 
reported fair or poor health were five times more likely to have widespread pain than women who reported no pain. In addition, those who were overweight, had depressive symptoms, a history of back pain or disability were three to four times more likely to have widespread pain than their peers without pain (Table 3 ). In men, only two factors were independently associated with widespread pain: radiographic polyarticular osteoarthritis and history of back pain. Men with these health conditions were about three to four times more likely to have widespread pain compared to other men (Table 3).

In a final set of analyses, we examined sex differences in likelihood for having widespread pain in women compared to men. Women had a three-fold increased odds for widespread pain compared to men and there was little change in the odds ratio after adjusting for several potential confounders including age, body mass index, hand OA, knee OA, depressive symptoms, fair or poor self-rated health, history of back pain, and disability (Table 4).

\section{Discussion}

Musculoskeletal pain was very common among elderly participants of the Framingham Study, reported by nearly two-thirds of women and half of men, aged 72 and older. These rates are similar to those reported in other studies of older populations (Centers for Disease Control, 2002; Lichtenstein et al., 1998). Sex differences in prevalence of pain were observed primarily in the proportions with widespread pain or no reported pain. Widespread pain was found in 1 out of 7 older women and 1 out of 20 older men in the elderly Framingham population. Slightly less than half of women and men had regional or multisite pain.

Widespread pain is poorly understood in the older population. Much of the research addressing the problem of widespread pain has been conducted in younger cohorts and often has been studied as a key symptom offibromyalgia. Forseth and colleagues studied a female population aged 20-49 years in Norway over a 51/2 year period and found that persons who had regional or multisite pain at baseline were more likely to develop widespread pain during follow-up than their peers without pain at baseline (Forseth et al., 1999). The authors proposed that widespread pain represents a continuum of chronic pain conditions and chronic pain in any site increases the risk for developing chronic widespread pain. A similar progression of pain over time was observed in a Swedish cohort aged 20-74 years, and persons aged 59-74 had the highest risk of developing widespread pain during the 3 year follow-up (Bergman et al., 2002). Our data showed that both men and women with widespread pain were more likely to have a history of back pain before age 65 than their peers without pain, suggesting that the progression of pain continues well into late life.

Sex differences in pain reporting have been attributed to a broad range of factors. Turk has proposed a biopsychosocial model of chronic pain that attributes sex differences in pain to interactions between biological, psychological, and sociocultural factors (Fillingim, 2003; Turk and Okifuji, 2002). In a sample of adults living in Kansas, Wolfe and colleagues found that women with and without fibromyalgia had a lower pain threshold using dolorimetry and had more tender points than men (Wolfe et al., 1995a). This heightened pain sensitivity among women, reported in a number of studies, is often used to explain greater reports of pain by women compared to men (Rollman and Lautenbacher, 2001). In the elderly, the problem of pain is more complex due to multiple comorbidities and, regardless of pain, the older population is more heterogeneous with regards to health and functioning. However, our findings show that the observed sex differences in widespread pain prevalence are substantial even in the oldest old and that adjusting for several potential confounders of this association, including age, arthritis, obesity, depression, self-rated health, disability, and pain history, did not alter the odds ratio showing that older women were three times more likely than older men to have widespread pain. 
The constellations of pain that we studied were shown previously to predict disability and falls in older women (Leveille et al., 2001, 2002). Number of pain locations is an important contributor to disability in older adults and older women report more locations of pain than older men (Scudds and Robertson, 2000). In the San Antonio Longitudinal Study of Aging, pain location was more strongly associated with functional limitations than either pain intensity or frequency (Lichtenstein et al., 1998). Although more work is warranted in this area, the findings to date underscore the need for comprehensive pain assessment that captures constellations of pain, in both clinical and research efforts involving older adults.

There were striking sex differences in the factors associated with widespread pain in the Framingham population. For example, polyarticular radiographic osteoarthritis (hand and knee OA) was strongly associated with widespread pain in men but not in women. This was interesting given that women generally had a somewhat higher prevalence of polyarticular hand and knee OA than men across categories of pain. The exception to this was in the group with widespread pain, where $56 \%$ of men and $45 \%$ of women had radiographic hand and knee OA (see Table 2). It is possible that in men, OA may explain more cases of widespread pain than in women. However, the fact that approximately half of men and women with widespread pain did not have radiographic OA of the hands and knees suggests that other pathological conditions underlie the problem of widespread pain in many older adults.

There are a few considerations in understanding our study results. It is likely that the prevalence of pain that we observed was an underestimate of the prevalence in the entire study cohort because older and more disabled persons, including those residing in nursing homes, were more likely to have missing pain information. We know from the WHAS, a population-based study of disabled older women, that one-quarter of participants had widespread pain, in contrast with $15 \%$ of women in the Framingham population (Leveille et al., 2001). Although joint pain is the most common pain in elders, the pain assessment was limited to musculoskeletal joint areas, thus missing pain in non-articular sites such as the thigh or lower leg.

Another limitation of this study is the limited sample size of older men, particularly those with widespread pain. This may have limited our ability to detect statistically significant trends or associations between potential risk factors and widespread pain in older men because of limited statistical power. In addition, because the Framingham Study was not designed to study the problem of chronic pain, we were unable to examine a number of other psychosocial and pain history factors that could contribute to chronic pain conditions in late life. Nevertheless, several associations were observed in these data and differences in musculoskeletal pain between men and women were evident.

In conclusion, our study presents new findings portraying sex differences in prevalence of musculoskeletal pain and associated risk factors. Pain was not only more prevalent but also more widespread in older women compared with older men. The differences between women and men in factors associated with pain were most notable in those with widespread pain. Further investigation of these differences is warranted and may uncover differing underlying pathology, indicating potentially different approaches to management. For now, however, we know that pain assessment restricted to selected common sites of pain such as the knees or hips in older patients may miss an important condition, widespread pain, which has profound accompanying risk factors that set it apart from other classifications of musculoskeletal pain.

\section{Acknowledgements}

This work was supported by the following: Arthritis Investigator Award from the Arthritis Foundation, National Institutes of Health No. AR47785, and National Heart, Lung and Blood Institute's Framingham Heart Study (Contract No. N01-HC-25195). 


\section{References}

Bergman S, Herrstrom P, Jacobsson LT, Petersson IF. Chronic widespread pain: a three year followup of pain distribution and risk factors. J Rheumatol 2002;29:818-25. [PubMed: 11950027]

Centers for Disease Control. Prevalence of self-reported arthritis or chronic joint symptoms among adults —United States, 2001. MMWR Morb Mortal Wkly Rep 2002;51:948-50. [PubMed: 12437034]

Croft P, Rigby AS, Boswell R, Schollum J, Silman A. The prevalence of chronic widespread pain in the general population. J Rheumatol 1993;20:710-3. [PubMed: 8496870]

Edmond SL, Felson DT. Prevalence of back symptoms in elders. J Rheumatol 2000;27:220-5. [PubMed: 10648042]

Felson DT, Kiel DP, Anderson JJ, Kannel WB. Alcohol consumption and hip fractures: the Framingham Study. Am J Epidemiol 1988;128:1102-10. [PubMed: 3189283]

Felson DT, Zhang Y, Hannan MT, Kannel WB, Kiel DP. Alcohol intake and bone mineral density in elderly men and women. The Framingham Study. Am J Epidemiol 1995a;142:485-92. [PubMed: 7677127]

Felson DT, Zhang Y, Hannan MT, Naimark A, Weissman BN, Aliabadi P, Levy D. The incidence and natural history of knee osteoarthritis in the elderly. The Framingham Osteoarthritis Study. Arthritis Rheum 1995b;38:1500-5. [PubMed: 7575700]

Fillingim RB. Sex, gender and pain: the biopsychosocial Model in action XX vs. XY: The International Journal of Sex Differences in the Study of Health. Dis Aging 2003;1:98-101.

Folstein MF, Folstein SE, McHugh PR. Mini-mental state: a practical method for grading the cognitive state of patients for the clinician. J Psychiatric Res 1975;12:189-98.

Forseth KO, Forre O, Gran JT. A 5.5 year prospective study of self-reported musculoskeletal pain and of fibromyalgia in a female population: significance and natural history. Clin Rheumatol 1999;18:114-21. [PubMed: 10357115]

Helme RD, Gibson SJ. The epidemiology of pain in elderly people. Clin Geriatr Med 2001;17:417-31 v. [PubMed: 11459713]

Hughes SL, Dunlop D, Edelman P, Chang RW, Singer RH. Impact of joint impairment on longitudinal disability in elderly persons. J Gerontol 1994;49:S291-S300. [PubMed: 7963286]

Jordan J, Luta G, Renner J, Dragomir A, Hochberg M, Fryer J. Knee pain and knee osteoarthritis severity in self-reported task specific disability: the Johnston County Osteoarthritis Project. J Rheumatol 1997;24:1344-9. [PubMed: 9228135]

Leveille SG, Guralnik JM, Ferrucci L, Hirsch R, Simonsick E, Hochberg MC. Foot pain and disability in older women. Am J Epidemiol 1998;148:657-65. [PubMed: 9778172]

Leveille SG, Ling S, Hochberg MC, Resnick HE, Bandeen-Roche KJ, Won A, Guralnik JM. Widespread musculoskeletal pain and the progression of disability in older disabled women. Ann Intern Med 2001;135:1038-46. [PubMed: 11747382]

Leveille SG, Bean J, Bandeen-Roche K, Jones R, Hochberg M, Guralnik JM. Musculoskeletal pain and risk for falls in older disabled women living in the community. J Am Geriatr Soc 2002;50:671-8. [PubMed: 11982667]

Lichtenstein M, Dhanda R, Cornell J, Escalante A, Hazuda H. Disaggregating pain and its effects on physical functional limitations. J Gerontol: Med Sci 1998;53A:M361-M71.

McAlindon TE, Cooper C, Kirwan JR, Dieppe PA. Determinants of disability in osteoarthritis of the knee. Ann Rheum Dis 1993;52:258-62. [PubMed: 8484690]

Mobily P, Herr K, Clark M, Wallace R. An epidemiologic analysis of pain in the elderly. J Aging Heath 1994;6:139-54.

Odding E, Valkenburg HA, Algra D, Vandenouweland FA, Grobbee DE, Hofman A. Associations of radiological osteoarthritis of the hip and knee with locomotor disability in the Rotterdam Study. Ann Rheum Dis 1998;57:203-8. [PubMed: 9709175]

Odding E, Valkenburg HA, Stam HJ, Hofman A. Assessing joint pain complaints and locomotor disability in the Rotterdam study: effect of population selection and assessment mode. Arch Phys Med Rehabil 2000;81:189-93. [PubMed: 10668773]

Radloff L. The CES-D scale: a self report depression scale for research in the general population. Appl Psych Meas 1977;1:385-401. 
Riley JL III, Robinson ME, Wise EA, Price DD. A meta-analytic review of pain perception across the menstrual cycle. Pain 1999;81:225-35. [PubMed: 10431710]

Rollman GB, Lautenbacher S. Sex differences in musculoskeletal pain. Clin J Pain 2001;17:20-4. [PubMed: 11289085]

Scudds R, Robertson M. Empirical evidence of the association between the presence of musculoskeletal pain and physical disability in community-dwelling senior citizens. Pain 1998;75:229-35. [PubMed: 9583758]

Scudds R, Robertson J. Pain factors associated with physical disability in a sample of communitydwelling senior citizens. J Gerontol A Biol Sci Med 2000;55:M93-M9.

Turk DC, Okifuji A. Psychological factors in chronic pain: evolution and revolution. J Consult Clin Psychol 2002;70:678-90. [PubMed: 12090376]

Vasan RS, Larson MG, Leip EP, Evans JC, O’Donnell CJ, Kannel WB, Levy D. Impact of high-normal blood pressure on the risk of cardiovascular disease. N Engl J Med 2001;345:1291-7. [PubMed: 11794147]

Wolfe F, Smythe H, Yunus M, Bennett R, Bombatdier C, Goldenberg D, Tugwell P, Campbell S, Abeles $\mathrm{M}$, Clark P, et al. The American college of rheumatology criteria for the classification of fibromyalgia. Report of the multicenter criteria committee. Arthritis Rheum 1990;33:160-72. [PubMed: 2306288]

Wolfe F, Ross K, Anderson J, Russell IJ. Aspects of fibromyalgia in the general population: sex, pain threshold, and fibromyalgia symptoms. J Rheumatol 1995a;22:151-6. [PubMed: 7699662]

Wolfe F, Ross K, Anderson J, Russell IJ, Hebert L. The prevalence and characteristics of fibromyalgia in the general population. Arthritis Rheum 1995b;38:19-28. [PubMed: 7818567]

Zhang Y, Xu L, Nevitt MC, Aliabadi P, Yu W, Qin M, Lui LY, Felson DT. Comparison of the prevalence of knee osteoarthritis between the elderly Chinese population in Beijing and whites in the United States: the Beijing Osteoarthritis study. Arthritis Rheum 2001;44:2065-71. [PubMed: 11592368]

Zhang Y, Xu L, Nevitt MC, Niu J, Goggins JP, Aliabadi P, Yu W, Lui LY, Felson DT. Lower prevalence of hand osteoarthritis among Chinese subjects in Beijing compared with white subjects in the United States: the Beijing Osteoarthritis Study. Arthritis Rheum 2003;48:1034-40. [PubMed: 12687546] 


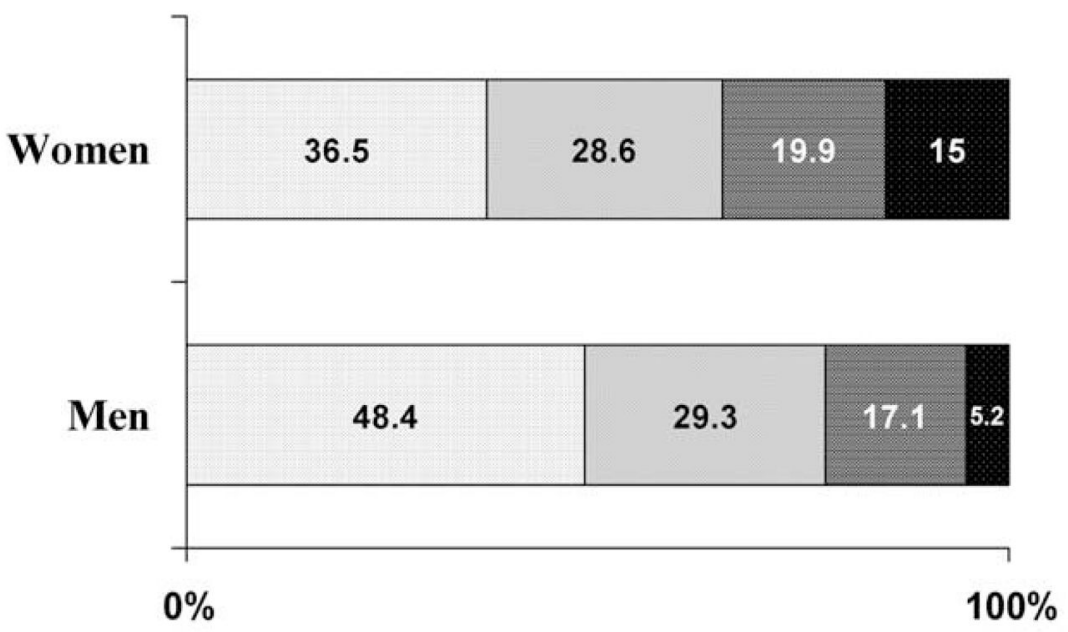

\section{no pain $\square 1$ region $\square 2-3$ regions}

widespread

Fig. 1.

Age-adjusted prevalence of musculoskeletal pain in women and men aged 72 years and older, Framingham, 1992-1993. 
Table 1

Characteristics of persons with non-missing and missing pain information, exam 22, Framingham Study, 1992-1993

\begin{tabular}{|c|c|c|}
\hline Characteristic & Non-missing, $\%(n=1062)$ & Missing, \% $(n=104)$ \\
\hline Age $>85$ years & 16.9 & $55.8^{* * *}$ \\
\hline Female & 64.2 & $76.0^{*}$ \\
\hline In-clinic exam ${ }^{a}$ & 86.5 & $20.2^{* * *}$ \\
\hline MMSE $<24^{b}$ & 30.1 & $79.8^{* * *}$ \\
\hline Radiographic knee arthritis & 40.1 & 42.7 \\
\hline ADL or mobility disability & 28.8 & $83.7^{* * *}$ \\
\hline
\end{tabular}

Chi-square test for between group differences,

* $P$-value $<0.05$

** $P$-value $<0.01$;

$* * *$ P-value $<0.001$.

${ }^{a}$ Alternative sites for exams for those with missing pain information were nursing homes (63\%), participants' homes (14\%) and other institutions (2\%).

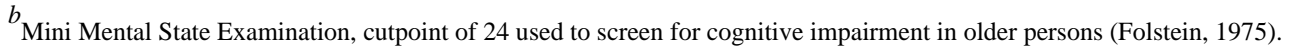


Leveille et al.

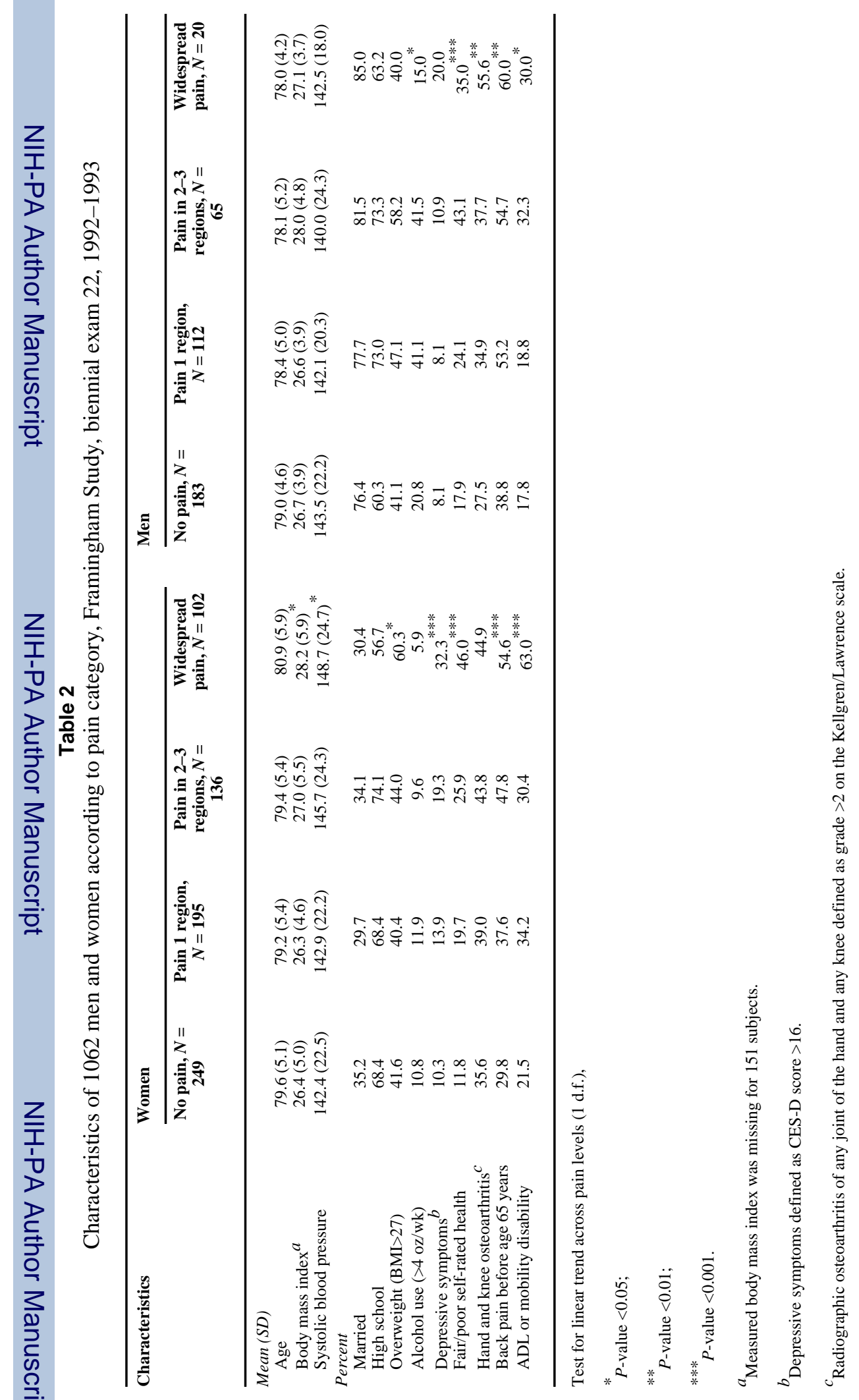

Pain. Author manuscript; available in PMC 2008 September 15. 


\section{Table 3}

Risk factors associated with widespread pain, Framingham Study, 1992-1993

\begin{tabular}{|c|c|c|c|c|}
\hline & \multicolumn{4}{|c|}{ Odds ratio $(95 \% \mathrm{CI})^{a}$} \\
\hline & \multicolumn{2}{|l|}{ Women } & \multicolumn{2}{|l|}{ Men } \\
\hline & No pain & Widespread pain & No pain & Widespread pain \\
\hline Age $>80$ years & 1.0 & $0.79(0.40,1.56)$ & 1.0 & $0.38(0.11,1.28)$ \\
\hline Overweight (BMI>27) & 1.0 & $3.66(1.78,7.53)$ & 1.0 & $0.56(0.17,1.87)$ \\
\hline Depressive symptoms $b$ & 1.0 & $3.19(1.44,7.07)$ & 1.0 & $3.26(0.76,13.95)$ \\
\hline Fair or poor self-rated health & 1.0 & $4.98(2.35,10.56)$ & 1.0 & $1.75(0.54,5.75)$ \\
\hline Hand and knee osteoarthritis ${ }^{c}$ & 1.0 & $1.46(0.76,2.78)$ & 1.0 & $3.75(1.27,11.07)$ \\
\hline Back pain < age 65 & 1.0 & $3.54(1.87,6.71)$ & 1.0 & $2.87(1.01,8.14)$ \\
\hline Disability $d$ & 1.0 & $3.27(1.58,6.76)$ & 1.0 & $1.35(0.37,4.91)$ \\
\hline
\end{tabular}

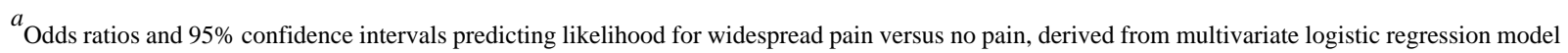
including all terms shown in table; model restricted to persons with no pain or widespread pain.

${ }^{b}$ Depressive symptoms defined as CES-D score $>16$.

${ }^{c}$ Radiographic osteoarthritis of any joint of the hand and any knee defined as grade $>2$ on the Kellgren/Lawrence scale.

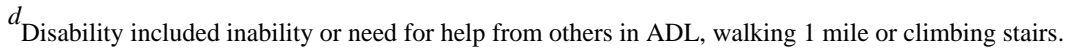


Table 4

Odds ratio predicting likelihood for widespread pain in women compared to men, adjusted for covariates, Framingham Study, 1992-1993

\begin{tabular}{lll}
\hline & Odds ratio (95\% CI) & Widespread pain, $\boldsymbol{n}=\mathbf{1 2 2}$ \\
\cline { 2 - 3 } & None or other pain, $\boldsymbol{n}=\mathbf{9 4 0}$ & $3.17(1.93,5.20)$ \\
\hline Women (versus men), unadjusted & 1.0 & $3.06(1.85,5.07)$ \\
+ age and overweight (BMI> 27) & 1.0 & $2.81(1.68,4.71)$ \\
+ hand OA, knee OA, depressive symptoms & 1.0 & $2.99(1.76,5.10)$ \\
+ fair or poor self-rated health, history back pain before age 65, & 1.0 & \\
mobility or ADL disability & & \\
\hline
\end{tabular}

Odds ratios and $95 \%$ confidence intervals predicting likelihood for women versus men to have widespread pain in the entire cohort, derived from serial multivariate logistic regression models including all terms shown in row in addition to previous rows. 